\title{
Evidence for entropy maximisation in human free choice behaviour
}

Natalie Rens 1, $^{*}$, Gian Luca Lancia ${ }^{2,3,{ }^{*}, \text { Mattia Eluchans }}{ }^{2,3}$, Philipp Schwartenbeck ${ }^{4,5}$, Ross Cunnington ${ }^{6}$, Giovanni Pezzulo ${ }^{2}$

1. Queensland Brain Institute, The University of Queensland, St Lucia Queensland 4072, Australia

2. Institute of Cognitive Sciences and Technologies, National Research Council, Via S. Martino della Battaglia, 44, 00185 Rome, Italy

3. University of Rome "La Sapienza", Rome, Italy

4. Wellcome Centre for Human Neuroimaging, University College London, London, United Kingdom

5. Oxford Centre for Functional MRI of the Brain, Nuffield Department of Clinical Neurosciences, University of Oxford, Oxford, United Kingdom

6. School of Psychology, The University of Queensland, St Lucia Queensland 4072, Australia

*. These authors contributed equally to this work

\section{Corresponding author}

Giovanni Pezzulo

Institute of Cognitive Sciences and Technologies,

National Research Council,

00185 Rome, Italy

E-mail: giovanni.pezzulo@istc.cnr.it 


\begin{abstract}
The freedom to choose between options is strongly linked to notions of free will. Accordingly, several studies have shown that individuals demonstrate a preference for choice, or the availability of multiple options, over and above utilitarian value. Yet we lack a decision-making framework that integrates preference for choice with traditional utility maximisation in free choice behaviour. Here we test the predictions of an inference-based model of decision-making in which an agent actively seeks states yielding entropy (availability of options) in addition to utility (economic reward). We designed a study in which participants freely navigated a virtual environment consisting of two consecutive choices leading to reward locations in separate rooms. Critically, the choice of one room always led to two final doors while, in the second room, only one door was permissible to choose. This design allowed us to separately determine the influence of utility and entropy on participants' choice behaviour and their self-evaluation of free will. We found that choice behaviour was better predicted by an inference-based model than by expected utility alone, and that both the availability of options and the value of the context positively influenced participants' perceived freedom of choice. Moreover, this consideration of options was apparent in the ongoing motion dynamics as individuals navigated the environment. In a second study, in which participants selected between rooms that gave access to three or four doors, we observed a similar pattern of results, with participants preferring the room that gave access to more options and feeling freer in it. These results suggest that free choice behaviour is well explained by an inference-based framework in which both utility and entropy are optimised and supports the idea that the feeling of having free will is tightly related to options availability.
\end{abstract}

Keywords: sequential choice; entropy maximization; free will; active inference 


\section{Introduction}

The ability to choose is considered central to libertarian theories of free will, which propose that decisions are experienced as free only when an individual has the 'ability to do otherwise' (Banja, 2015; Roskies, 2010; Stillman et al., 2011). This preference for choice, in which individuals preferentially select options that provide further choices, has also been observed in numerous behavioural studies (Bown et al., 2003; Cockburn et al., 2014; Fujiwara et al., 2013; Leotti and Delgado, 2011; Ono, 2000). When faced with choosing between an option that leads to a set action or a an option that leads to a subsequent choice, humans, and other species, show a preference for the option providing further choice (Bown et al., 2003; Catania, 1975; Fujiwara et al., 2013; Leotti and Delgado, 2011; Ono, 2000; Ryan and Deci, 2000; Suzuki, 1999, 1997). Individuals are also more likely to choose an item when offered with an alternative than when the same item is offered alone (Bown et al., 2003). This behaviour is evident despite the additional choice providing no gain in reward, or in some cases, even a sacrifice of reward (Bennett et al., 2016). This "over-valuation" of choice seems paradoxical when considered according to utilitarian value alone, and has led to the suggestion that the ability to choose may be rewarding in itself, serving to encourage an individual to exercise control over the environment (Fujiwara et al., 2013; Inesi et al., 2011; Leotti et al., 2010; Leotti and Delgado, 2011).

As choice availability provides no utility of its own, choice preference has been difficult to account for with classical economic decision models that consider decisions on the basis of expected utility alone (Kahneman and Tversky, 1979; Nash, 1950). The value of choice is only considered when it increases the likelihood of attaining a preferred item, such as when the likelihood of a preferred item being available is increased by having a larger option set (Pattanaik and $\mathrm{Xu}, 1998$ ). However, it does not account for an inherent preference for choice when it does not affect the value of the outcome attained. Recently, inferential accounts of decision-making have considered the value of occupying states with more options available as a means of entropy maximisation, in addition to utility maximisation, and may, therefore, better account for the preference for choice.

Inferential accounts of decision-making are based on the idea that decision and planning problems are accomplished using probabilistic inference, with an internal model that represents the contingencies between actions and outcomes. These include active inference (Friston et al., 2017, 2015; Pezzulo et al., 2016; Schwartenbeck et al., 2018, 2015), probabilistic planning-as-inference (Botvinick and Toussaint, 2012; Pezzulo and Rigoli, 2011; Solway and Botvinick, 2012; Stoianov et al., 2018) and KL control (Kappen et al., 2012). As a predominant example in human cognition, active inference posits that the brain uses approximate Bayesian inference to steer both perception and action planning (Friston, 2010; Parr et al., 2022; Pezzulo et al., 2015). Action selection is described as a process of minimization of a measure of discrepancy between an agent's expectations and its observations. Under some assumptions, this measure of discrepancy can be assimilated to "surprise" or "prediction error", but more formally it is defined as a "free energy" and hence active inference agents select actions that (are expected to) minimize free energy. Crucially, the process of free energy minimization is different from utility maximization, since it considers utility and entropy contributions to choice; see the Methods and (Parr et al., 2022) for more details.

The entropy component suggests that, in the absence of precise prior beliefs, maximally uncertain beliefs about the hidden states of the world should be adopted. This corresponds to Jaynes' maximum entropy principle, which states that the probability distribution which best represents the current state of knowledge about a system is the one with largest entropy (Jaynes, 1957). In the context of decision-making, this implies that an agent should actively seek higher entropy states that offer more future choices, or "keep its options open" (Klyubin et al., 2008). This is because maintaining highly precise prior beliefs in uncertain settings can likely produce large prediction errors; conversely, by maximising the number of expected possibilities and the entropy of its prior beliefs, the agent can minimise its future surprise (or more formally, free energy) (Parr et al., 2022; Pezzulo and Ognibene, 2011; Schwartenbeck et al., 2015). In sum, in comparison to utility maximisation, inference-based models cast both utilitarian preferences and the maximisation of options within one and the same adaptive mechanisms for decision-making (Friston, 2010; Friston et al., 2017; Pezzulo et al., 2018; Pezzulo et al., 2015), and could offer a framework to 
explain free choice behaviour. Importantly, models based on expected utility alone versus inference-based models that additionally account for entropy can lead to divergent predictions about choice behaviour, hence these alternative predictions remain to be tested empirically. Furthermore, given the conceptual link between the 'ability to do otherwise' in free will and preference for choice, a novel consideration is that states of higher entropy could correspond to greater perceived freedom of choice.

The objectives of this work were twofold. The first was to investigate whether entropy maximization is a significant consideration in guiding sequential choice behavior, as predicted by inference-based schemes. The second objective was to test the hypothesis that states with greater utility and entropy would be subjectively experienced with higher perceived freedom of choice, as reported by participants. For this, we designed two two-step decision studies in a virtual environment, where participants freely navigated through two consecutive rooms to find rewards. In the first study (Experimental task 1), participants learned that choosing one room led to two doors that were always open while, in the second room, one of the doors was always locked. The location of the locked door in the second room changed trial to trial. This was essential to ensure that participants could choose, or sample, between four outcomes overall but that one room was always associated with more options (higher entropy) than the other. By manipulating the relative values of reward outcomes between the two rooms, we were able to determine the relative influence of value and entropy on participants' choice behaviour. We hypothesised that choice behaviour would be better explained by a model predicting a bias towards the room providing both more choice and higher reward, than a pure expected utility model, predicting a bias towards the room with higher reward alone. We further predicted that both these factors of utility and entropy would influence participants' perceived sense of freedom. We also analysed the participants' motion dynamics as they navigated the virtual environment for evidence of ongoing choice deliberation. We hypothesised that motion trajectory variability and changes of mind would be greater in the context of greater choice availability, reflecting the consideration of alternative choice options throughout the decision-making period. Finally, we designed a second study (Experimental task 2) with the same structure as the first study, but in which participants selected between a room with four doors always open versus a room with one door closed. This second study permits assessing the generality of a putative 'preference for choice', beyond the case of one versus two rooms.

\section{Materials and Methods}

\section{Experimental task 1: decision between 2-Choice vs. 1-Choice rooms}

\section{Participants}

Twenty-eight participants were recruited from the University of Queensland after providing written informed consent. All participants were right-handed, had normal or corrected to normal vision, and no history of psychiatric disease, neurological trauma or disorder. The only exclusion criteria were that participants be of at least 18 years of age. One participant was excluded for failing to properly follow task instructions, and data from the remaining 27 participants were used for the behavioural analyses (23 female, mean age 21 years, range 18-24 years). Participants were monetarily compensated for their time. The University of Queensland Medical Research Ethics Committee approved the experiment.

\section{Task design}

The task was based in a virtual environment, presented using Psychtoolbox on MATLAB (MathWorks, Inc., Natick, MA, USA). Participants viewed a first-person perspective from their current position in a three-dimensional environment, which updated continuously as they moved. The paradigm of the two-step decision task is shown in Figure 1. Participants received instructions 
and underwent a training period to practise navigating in the task environment prior to commencing the experimental task. Participants were asked to make two consecutive decisions between two sets of doors (one set of doors for each decision) in order to reach the location of gems they would like to collect. To navigate the environment, participants held down the up-arrow key to move forward and the left and right arrow keys to turn in each direction. When a door was reached, it could be opened by pressing the space bar. Choosing the left or right door in the first room would determine the subsequent room, and choosing left or right in the second room would open the door to receive the gem at that location.

Critically, participants were informed that a final door could be "barricaded" (locked), in which case it was unavailable to choose on that trial and the other available door should be selected. To aid in the training, selecting the locked door resulted in a skull cue with no points gained. Participants rapidly learned not to select this door during the training period, which was confirmed by no participant choosing this door during the experimental task. In each block, one of the final rooms always had one locked door. The location of this room remained constant throughout each block, enabling participants to quickly differentiate the two rooms, but the specific locked door (left or right) changed pseudo-randomly on each trial. Hence, in the first room, one door always led to a room that had both doors available (2-Choice), whereas the other door always led to a room that had only one door available (1-Choice). Importantly, this design ensured that four final locations were available throughout the block, such that if participants attempted to equally sample each location the probability of choosing each of the two final rooms would be $0.5(2 / 4)$; see Figure 1 .

A gem, of varying value, was gained after selection of any available door and presented for $1 \mathrm{~s}$. Within each room, the mean value of the gems behind both doors was equivalent, but the actual number of points received each trial varied according to a set variance of 2 points. The participants were informed that "Each pile of gems is worth a certain number of points", and that their locations would remain consistent throughout each block. Participants were not informed of the precise values but were told that the points gained from a pile could vary between trials. Critically, the relative values of the gems between the two rooms varied between blocks according to five conditions. In the first condition, the mean values of gems in each room were equal ( $M=6$ points).

This condition permitted us to test if there was any choice bias present for each participant when values of rewards were equal across all doors. If the availability of choice was a factor in participants' choices, we would expect the choice proportion for the 2-Choice room to be greater than 0.5 , whereas equal probability would indicate choices were not influenced by the number of options. In two conditions, the value of gems in the 2-Choice room was either greater ( $M=8$ points) or much greater ( $M=10$ points) than the value of the gems in the 1-Choice room (M=6 points). In the remaining two conditions, the reverse was true. This factorial design enabled us to investigate the combined effects of option availability and value on choice behaviour. Before the experiment, the participants made 10 practice trials. During the experiment, two blocks of each of the five conditions were presented, counter-balanced for door position, and each block consisted of 40 trials, giving 80 trials in total for each condition.

Following each block, participants provided a rating of their perceived level of freedom for the left and right rooms on a free-sliding visual analogue scale with 'Not free' $(0)$ at one end and 'Very free' at the other (10) (Filevich et al., 2013). The slider was centred (5), requiring participants to use the left arrow to indicate less free and the right arrow to indicate more free. Participants were instructed to give this rating according to their own interpretation of "how free" they felt their decision for each room was. The room positions were counterbalanced in order to circumvent stereotypical and automated responding, but allowed us to determine the perceived freedom for each room. 


\section{2-Choice 1-Choice}
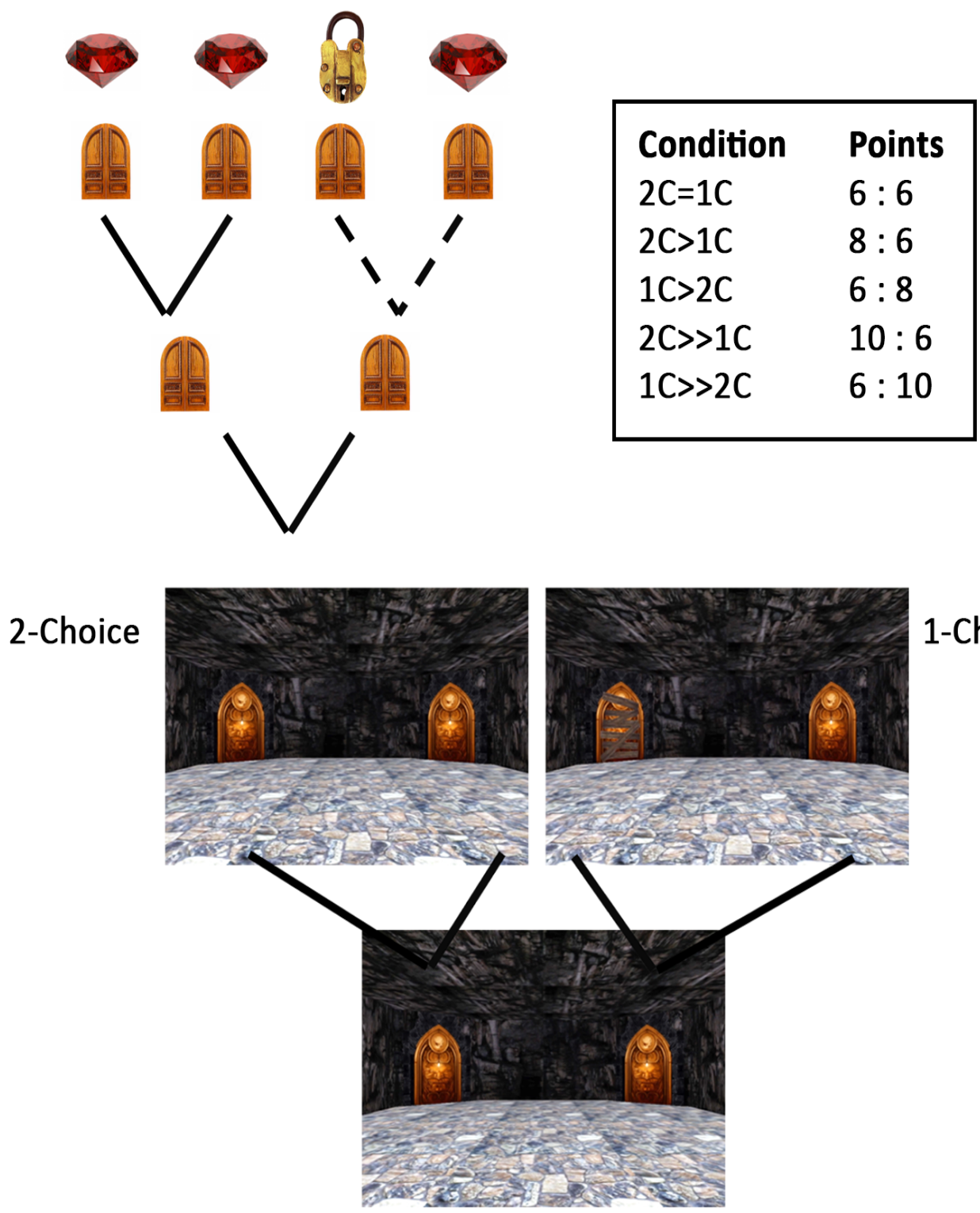

1-Choice

Figure 1. Design of Experimental task 1. Participants were instructed to navigate the virtual maze environment in order to reach the gem that they wished to choose. Left panel shows the decision tree for a single trial. Participants first chose between two doors leading to two separate rooms. In one room, both doors were always available (2-Choice), whereas in the second room one of the doors (randomly left or right) was always locked (1-Choice). The value of the gems was manipulated so that the two rooms were either equivalent in value or one was comparatively more valuable than the other. Right panel shows the participant perspective for the doors in each of the rooms, noting that the only difference is the presence of a barricade across the left door in the 1-Choice room on the right-hand side.

\section{Behavioural analysis}

We calculated choice bias by the proportion of trials the 2-Choice room was selected over the 1-Choice room. We performed a one-sample t-test to determine if the bias was significantly different from 0.5 for the baseline condition $(2 C=1 C)$ and also overall. We also quantified the choice bias of each participant, by considering whether the proportion of times they selected the 2Choice room was greater than 0.5. Furthermore, we carried out a linear regression analysis to ascertain to what extent individual participant choices were predicted by expected utility. For each trial, utility was calculated as the relative reward value of the gems in the 2-Choice room compared 
to the 1-Choice room, which changed in each condition according to the respective mean gem value of each room, for example +2 in the $2 \mathrm{C}>1 \mathrm{C}$ condition and -2 in the $1 \mathrm{C}>2 \mathrm{C}$ condition. The dependent variable was choice response, which we coded as +1 for the 2 -Choice room and -1 for the 1-Choice room on each trial. A positive coefficient would indicate a positive influence of each factor, whereas a negative coefficient would signify avoidance. We assessed group-level significance with one-sample t-tests for utility and entropy gain.

Subjective reports of perceived freedom of choice for each room were analysed with a $2 \times 5$ repeated measures ANOVA, including factors of Choice Availability (1-Choice or 2-Choice rooms) and Value, separated by the five conditions from the lowest to highest comparative value for each room. For Value, Mauchly's Test of Sphericity indicated that the assumption of sphericity had been violated $(p<0.001)$, so a Greenhouse-Geisser correction was applied.

\section{Model specification}

We created a computational model of the task environment to compare predictions from a classical expected utility model (Kahneman and Tversky, 1979; Nash, 1950) with an active inference model (Friston et al., 2013; Pezzulo et al., 2015). Expected utility theory is based on the idea that agents maximise utility, but are otherwise insensitive to other features of the environment. In comparison, active inference predicts that agents are driven by probabilistic planning to maximise entropy as well as utility. Note that while we implement an active inference model, similar predictions can be made with other inference-based models that incorporate entropy. Below we briefly describe the active inference model (using a Markov Decision Process approach) as well as its reduced version that implements expected utility alone.

Active inference is built on the premise that an agent builds a generative model of its decision context, which creates a probabilistic mapping from the underlying hidden states to the outcomes that are observed. This mapping is updated using variational Bayes, described in detail in Friston et al. (Friston et al., 2017). The assumption is that, in order to plan upcoming choices, agents perform inference on three main elements: the current state, policies, and the precision of beliefs. The current state reflects a probabilistic belief about the state the agent is in. Policies are the possible action sequences an agent can take to reach (probabilistically) future states. Precision reflects the agent's confidence in policy selection, and it controls the stochasticity of action selection (Friston et al., 2017).

The generative model of an active inference model is specified by $A, B, C$ and $d$ matrices (Figure 2). The A matrix represents an agent's observations and provides the mappings from hidden states $\mathrm{s}$ to observations $\mathrm{o}$ at each transition $\mathrm{t}, P\left(o_{t} \mid s_{t}\right)$. In the simplified version of the task that we implemented, there are only four directly-observable states and each is uniquely associated with an outcome. The maze has a starting location, two states corresponding to two available reward locations possible in the 2-Choice room and one state corresponding to the single available reward location in the 1-Choice room. The $B$ matrix denotes the transition probabilities based on the current hidden state and the action taken a, $P\left(s_{t+1} \mid s_{t}, a_{t}\right)$. In this case, the agent makes only the first action to choose left or right, leading to the 2-Choice or 1-Choice room, respectively. The left policy had a $50 \%$ likelihood of the agent reaching either one of the two possible reward locations, whereas the right policy led deterministically to the one available reward, illustrating the fact that the 2-Choice room is associated with higher entropy over outcomes than the 1-Choice room. The $c$ and $d$ vectors correspond to an agent's prior beliefs about observations and initial states, respectively. We specified a c-vector for every condition, to account for the changing reward value of the gems ( $c=2,3$, or 4 equivalent to 6,8 , or 10 points).

We simulated agent behaviour according to either expected utility (equation 1) or an active inference scheme, which maximises both expected utility and entropy (equation 2).

$$
\begin{aligned}
& E(U)=\sum_{S_{T}} P\left(S_{T} \mid S_{t}, \pi\right) \cdot u\left(S_{T} \mid m\right) \\
& -D_{K L}\left[P\left(S_{T} \mid S_{t}, \pi\right) \| P\left(S_{T} \mid m\right]=H\left[P\left(S_{T} \mid S_{t}, \pi\right)\right]+\sum_{S_{T}} P\left(S_{T} \mid S_{t}, \pi\right) \cdot u\left(S_{T} \mid m\right)\right.
\end{aligned}
$$


The expected utility model considers the probability of reaching future states $S_{t}$ by following a given policy $\pi$, and the utility $u$ of these states. The inference model incorporates an additional factor of entropy $H$ over those future expected states (right side of equation 2). Predictions from each of these models were then compared to participant choice behaviour in order to determine the respective model evidence.

Hidden states

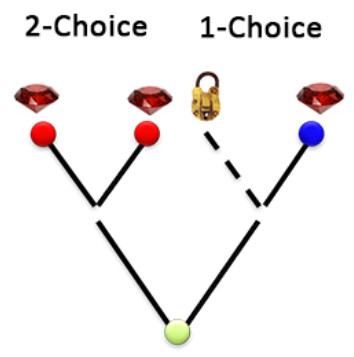

$$
\begin{gathered}
Y^{\prime} V^{\prime} V^{\prime} V^{\prime} \\
A:=\left[\begin{array}{llll}
1 & 0 & 0 & 0 \\
0 & 1 & 0 & 0 \\
0 & 0 & 1 & 0 \\
0 & 0 & 0 & 1
\end{array}\right]
\end{gathered}
$$

Transition probabilities

$$
B_{\text {left }}:=\left[\begin{array}{cccc}
0 & 0 & 0 & 0 \\
0.5 & 1 & 0 & 0 \\
0.5 & 0 & 1 & 0 \\
0 & 0 & 0 & 1
\end{array}\right] \vee_{V^{\prime}}^{\prime} \quad V_{\text {right }}^{\prime}:=\left[\begin{array}{llll}
0 & 0 & 0 & 0 \\
0 & 1 & 0 & 0 \\
0 & 0 & 1 & 0 \\
1 & 0 & 0 & 1
\end{array}\right] V^{\prime}
$$

Preferences

$$
\begin{aligned}
& C_{2 C=1 C}:=\left[\begin{array}{lll}
0 & 2 & 2
\end{array}\right] \\
& c_{2 C>1 C}:=\left[\begin{array}{llll}
0 & 3 & 3 & 2
\end{array}\right] \\
& \text { 13 } c_{1 C>2 C}:=\left[\begin{array}{llll}
0 & 2 & 2 & 3
\end{array}\right] \\
& c_{2 C \gg 1 C}:=\left[\begin{array}{llll}
0 & 4 & 4 & 2
\end{array}\right] \\
& c_{1 C \gg 2 C}:=\left[\begin{array}{lll}
0 & 2 & 2
\end{array}\right]
\end{aligned}
$$

Initial state

$$
d:=\left[\begin{array}{llll}
1 & 0 & 0 & 0
\end{array}\right] Y^{\prime}
$$

Figure 2. Specification of the generative model used for the computational analysis of Experimental task 1. The A matrix provides the mappings from hidden states to observations. In this model, there were only four states possible and each mapped to a unique outcome. The first state corresponds to the starting point, states 2 and 3 represent the reward locations possible in the 2-Choice room and state 4 is the single reward location in the 1-Choice room. The $B$ matrix defines the transition probabilities. The agent could choose left or right to select the 2-Choice or 1-Choice room, respectively. Choosing left led to a $50 \%$ probability of reaching either one of the two reward locations. Choosing right led deterministically to the single reward location. The c-vector was specified for each condition to represent a prior on the reward value of the gems available in each room. The $d$ vector denotes the prior belief of the initial state.

We performed model inversion, based on variational Bayes, using the spm_dcm mdp.m function within the DEM toolbox (SPM12, Wellcome Trust Centre for Neuroimaging, London, UK) (Schwartenbeck and Friston, 2016). Participant choices were coded as a Markov decision process, in which states corresponded to the left or right doors chosen in the 2-Choice room and the door chosen (regardless of location) in the 1-Choice room. Actions corresponded to choosing the 2Choice ("left") room or 1-Choice ("right") room. We obtained separate MAP-estimates for 
participants' priors on precision $(\beta$, where precision $=1 / \beta)$, which characterises a participant's tendency towards goal-directed behaviour, for each of the five conditions with a prior mean of 0 and a prior variance of $1 / 16$. Higher $\beta$ values indicate less influence of free energy in driving policy selection and, therefore, more randomness. After model inversion, we compared all free energies, which provide an approximation to model evidence, for each participant using Bayesian model comparison to determine whether participant behaviour was better predicted by expected utility or active inference (Rigoux et al., 2014; Stephan et al., 2009).

\section{Decision trajectory analysis}

The Cartesian $(\mathrm{x}, \mathrm{y})$ coordinates of the participant's position in virtual space were measured with every screen refresh $(60 \mathrm{~Hz})$ in order to determine the trajectories of participants' movements towards the doors for each decision. Approximately $2 \mathrm{~s}$ of data were collected for each room, according to the individual response time and trajectory. Our primary analysis concerned the trajectories in each of the final rooms (2-Choice and 1-Choice rooms), given our hypothesis that physical markers of deliberation would be greater when an alternative option remained available than when faced with only one option. For completion, we also analysed the trajectories in the first room, splitting trials according to which second room was chosen on that trial.

We first calculated trials that contained one or more reversals in direction that occurred along the $x$-axis in the trajectory, termed ' $x$-flips' (Dale et al., 2008). This measure of motion trajectory complexity is thought to arise due to competing evidence, or uncertainty, in the underlying decision plan (McKinstry et al., 2008). In contrast to typical mouse-tracking studies, the keyboard navigation in our computer virtual environment was relatively smooth, and so we used this measure only to examine gross changes in direction that occurred as an indication of change of mind behaviour.

We also measured a second, related, factor of decision variability using the discrete (Fréchet) distance (equation 3). This function measures the similarity between two curved trajectories to find the minimum distance that connects each line (Eiter and Mannila, 1994; Fréchet, 1906).

$$
D_{\text {Frechet }}(A, B)=\inf \max _{\alpha, \beta \in[0,1] t \in[0,1]}\left\{\|A(\alpha(t)), B(\beta(t))\|_{2}\right\}
$$

This is commonly referred to as the "walking-dog distance", as it can be understood as the minimum length of leash that would be required for a man walking his dog if each were on the separate paths (Eiter and Mannila, 1994; Fréchet, 1906). We first time-normalised each trial to 101 time-points and remapped the trajectories going to the left door to the right door (or vice versa), as common in mouse tracking studies (Freeman et al., 2011). We excluded from the analysis $x$-flip trials, as these would lead to abnormally large peaks in distance. We calculated an average trajectory for each participant, in each condition and room, and then, on each trial, calculated the discrete Fréchet distance between the trial trajectory and the average trajectory for the context. We expected that if the availability of an alternative option (the non-selected door) remained actively considered then this would result in greater variability in decision trajectories in the 2-Choice room than the 1-Choice room. Group differences in x-flips and Fréchet distance were determined using 2x5s ANOVA of Choice Availability and Value, organised from comparatively least valuable to most valuable for the chosen room on that trial. Two sets of $2 \times 5$ ANOVAs were performed, in order to compare trajectories first in the 1-Choice and 2-Choice Rooms, then in the first room, on the basis of whether the 1-Choice or 2-Choice room was chosen. 


\section{Experimental task 2: decision between 4-Choice vs. 3-Choice rooms}

\section{Participants}

Fifty participants were recruited online, using Prolific (www.prolific.co). The only exclusion criteria were that participants be of at least 18 years of age. Fourteen participants were excluded for failing to properly follow task instructions, and data from the remaining 36 participants were used for the behavioural analyses (16 female, mean age 21.8 years, range 18-24 years). Participants were monetarily compensated for their time. The Ethics committee of the National Research Council approved the experiment.

\section{Task design}

Experimental task 2 has the same design as Experimental task 1, but in the first room, one door always led to a room that had four doors available (4-Choice), whereas the other door always led to a room that had only one door closed (3-Choice). As in experimental task 1, one of the rooms (the 4-Choice room) is associated with higher entropy over outcomes than the other (3Choice) room and the closed door in the 3-Choice room changed on a trial-by-trial basis. The task was based on a virtual environment designed in Unity (www.unity.com) to be delivered online on mobile phones. The structure of the virtual environment was the same as Experimental task 1, but some graphical elements were different to be adapted to mobile phones and participants performed the task using a virtual analog controller, rather than a keyboard.

As in Experimental task 1, we included the two factors of Choice Availability and Value. Like Experimental task 1, we used two conditions of Choice Availability (4-Choice and 3-Choice). However, we only included three (rather than five) conditions of Value, to keep the online experiment shorter and ensure that data quality did not decrease over time. In the baseline condition $(4 C=3 C)$, the value of gems in the 4-Choice and the 3 -Choice rooms was the same $(M=6$ points). In another condition $(4 \mathrm{C}>>3 \mathrm{C}$ ) the value of the gems in the 4-Choice room was much greater ( $M=10$ points) than the value of the gems in the 3-Choice room. In the last condition $(3 C>>4 C)$, the reverse was true. Furthermore, selecting the locked door did not have any effect, rather than resulting in a skull cue. The position of rooms was counterbalanced across participants. This factorial design enabled us to investigate the combined effects of option availability and value on choice behaviour, when participants selected between a more options compared to Experimental task 1.

Before the experiment, the participants made 10 practice trials. During the experiment, three blocks were presented, one for each of the three experimental conditions, counter-balanced for door position, and each block consisted of 25 trials. Finally, as for experimental task 1, following each block, participants provided a rating of their perceived level of freedom for the left and right rooms.

\section{Behavioural analysis}

The behavioural analyses followed the same approach as Experimental task 1 and used 2x5 ANOVAs, with two levels of Choice Availability conditions (4-Choice and 3-Choice) and three Value conditions $(4 C=3 C, 4 C>>3 C$, and $3 C>>4 C)$. The significance level was set to $p<0.05$ for all the analyses. 


\section{Results}

\section{Results of Experimental task 1}

\section{Participant choice behaviour}

We first analysed choice proportions in favour of the 2-Choice room over the 1-Choice room across all five conditions of varying relative reward values. If there was no influence of option availability and participants equally chose each of the two rooms, choice proportions would be 0.5 when the two rooms were equally valuable. If participants exhibited a choice bias, the proportion in favour of the 2-Choice room would be $>0.5$. In the baseline condition, when all rewards were equal, we found that participants showed a highly significant choice bias, selecting the 2-Choice room on $59 \%(\mathrm{SE}=2 \%)$ of trials on average (single-sample $\mathrm{t}$-test difference from $50 \% \mathrm{t}(26)=3.909$, $p<0.001)$. This bias was further evident when all conditions were analysed collectively, resulting in an overall choice bias of $58 \%(\mathrm{SE}=1 \% ; \mathrm{t}(26)=6.028, \mathrm{p}<0.001)$. This is apparent in the positive shift in choice proportions above chance $(50 \%)$ towards the 2-Choice room, shown in Figure $3 \mathrm{~A}$.

To further examine whether and how many individual participants showed a positive choice bias, we plotted the proportion of times each participant selected the 2-Choice room, see Figure 3B. Overall, almost all the participants showed a positive choice bias, as indexed by a proportion greater than 0.5 , but they varied in the choice bias intensity.

Furthermore, we quantified the contributions of utility-seeking to each participant's choice behaviour, to ensure that participants were overall able to select rewarding outcomes. For this, we performed a linear regression analysis to predict participant's choices on each trial on the basis of comparative value. We found that expected utility significantly influenced behaviour (mean $\beta=0.10$, $\mathrm{SE}=0.02, \mathrm{t}(26)=6.070, \mathrm{p}<0.001)$. Individual $\beta$ coefficients for each participant are displayed in Figure $3 \mathrm{C}$. Positive coefficients in the regression models indicated that participant choice behaviour was positively driven by utility-seeking factors. We found that this was the case for almost all participants. Finally, we asked whether the drive for entropy gain led to a detriment in the reward gained by each participant. We correlated the choice bias for each participant with the $\beta$ coefficient for utility gain and found no relationship (Pearson $R=0.01, p=0.96$ ). Hence, entropymaximising behaviour was not associated with a trade-off in utilitarian gain. 

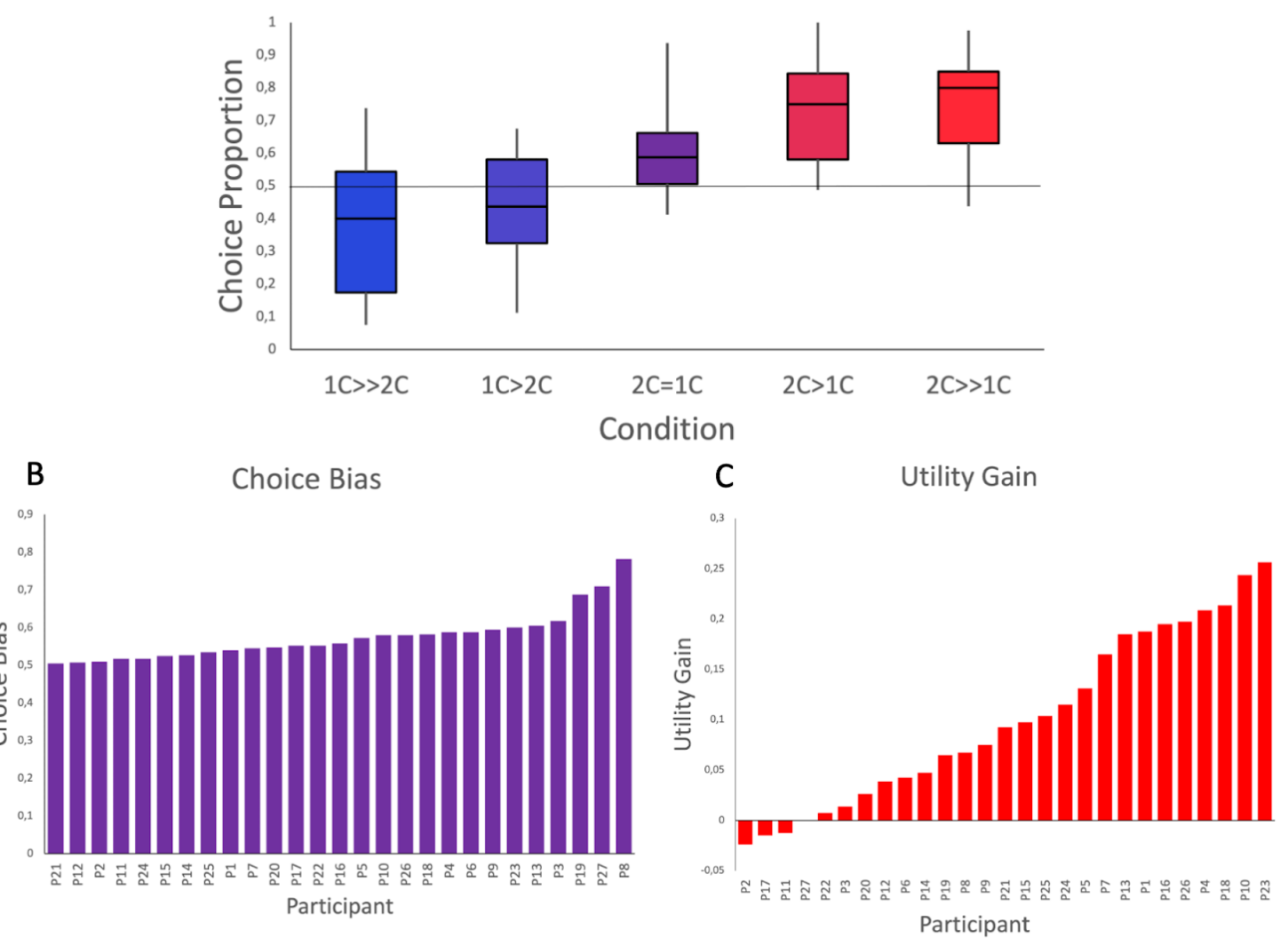

Figure 3. Participant choice behavior during Experimental task 1. A) Box-and-whisker plots show distributions of participant choice biases for each condition. Participants showed a significant choice bias, evidenced by a greater proportion of choices for the 2-Choice (2C) room than the 1-Choice (1C) room. This choice bias was present across all conditions, as seen by the positive shift in choice proportions. B) Individual choice bias, quantified by the proportion of choices of the 2-Choice room. Almost all participants showed a proportion greater than 0.5 , which indexes a positive choice bias. C) Individual linear regression coefficients for the degree to which each participant's choices were predicted by utility gain, sorted in ascending order. Positive coefficients indicate utility-seeking behaviour, whereas negative coefficients indicate avoidance. Participants varied in the extent but, overall, showed a positive tendency for utility.

\section{Model-based predictions}

We simulated a simplified Markov decision process model of the task environment in order to estimate expected behaviour according to expected utility and a representative inference-based method, active inference, which additionally incorporated entropy maximisation. We simulated only the first action of selecting between each of the rooms, because this is when the putative entropyseeking behaviour may manifest. In our simulations, choosing the 2 -Choice room gave a $50 \%$ likelihood of reaching each of two final outcomes, and the 1-Choice room led to one deterministic outcome.

The two models under scrutiny, utility maximisation and active inference, make opposing predictions, particularly when the value of two contexts is equal. Specifically, the utility maximisation model predicts no bias towards either room; whereas the active inference model predicts a probability of $58 \%$ for the 2 -Choice room. The latter prediction was a very close match to the average choice bias of $59 \%$ exhibited by participants in our study. Thus, the active inference model accounts well for the bias towards choice observed in our participants in this paradigm.

Furthermore, we performed model inversion of the participant choice responses according to expected utility and active inference models, respectively. Bayesian model comparison provided significant evidence to support an active inference model of free choice behaviour (Supplementary 
Tables 1 and 2 for individual $\beta$ parameters and free energy). We attained an exceedance probability $\varphi=1.0$ in favour of an active inference model or, applying a conservative correction, protected exceedance probability $\varphi=0.678$ (Figure 4). Hence, there was significant evidence that active inference better accounted for participant behaviour than expected utility alone.

\section{Model Comparison}

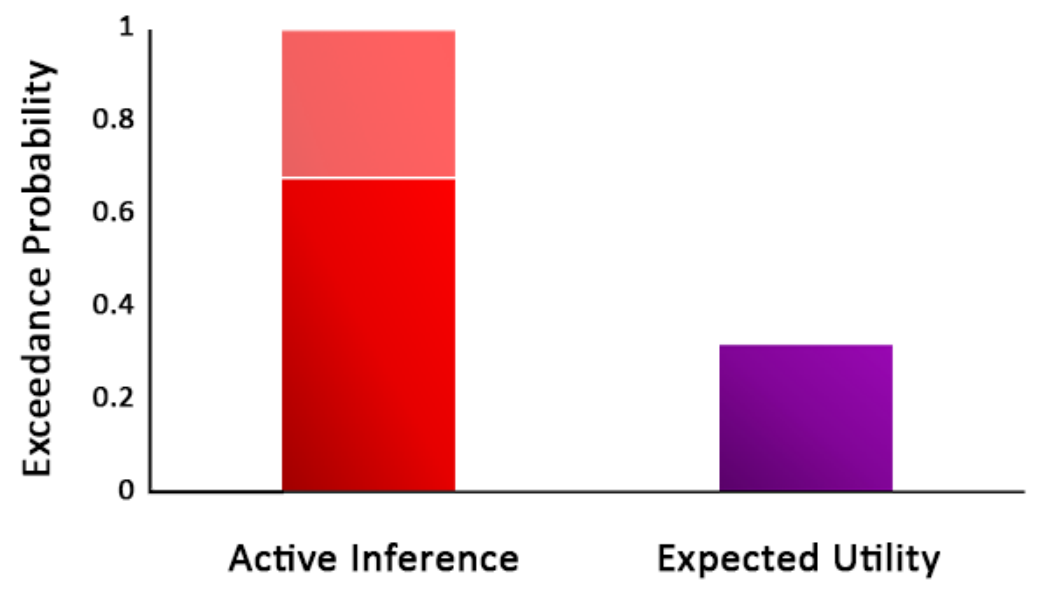

Figure 4. Random effects Bayesian model comparison provide evidence for an active inference model over expected utility alone. The exceedance probability for the active inference model was 1.0 (protected exceedance probability 0.678; darker red)

\section{Subjective free choice ratings}

Average ratings of perceived freedom of choice differed significantly across conditions (Figure 5). A $2 \times 5$ repeated measures ANOVA revealed a main effect of Choice Availability, in which choices made in the 2-Choice room were perceived as freer than those in the 1-Choice room (2Choice: $M=6.9, S E=0.3,1-C h o i c e: ~ M=5.4, S E=0.4 ; F(1,26)=10.843, p=0.003)$. Perceived freedom was neutral at minimum in the 1-Choice room and positively modulated by the availability of an alternative option in the 2- Choice room. There was also a main effect of Value, indicating that the reward available in each room had a positive influence on perceived freedom $(F(1.874,48.722)=5.224, p=0.01)$. There were no significant interactions. Note that for the statistical analysis, conditions were ordered according to increasing value of reward. Therefore, in Figure 5, the main effect of Value is apparent as increasing Free Ratings to the right across conditions for the 2-Choice room (i.e. as the relative value of the 2-Choice room increased) and, conversely, increasing Free Ratings to the left across conditions for the 1-Choice room (i.e. as relative value of the 1-Choice room increased). 


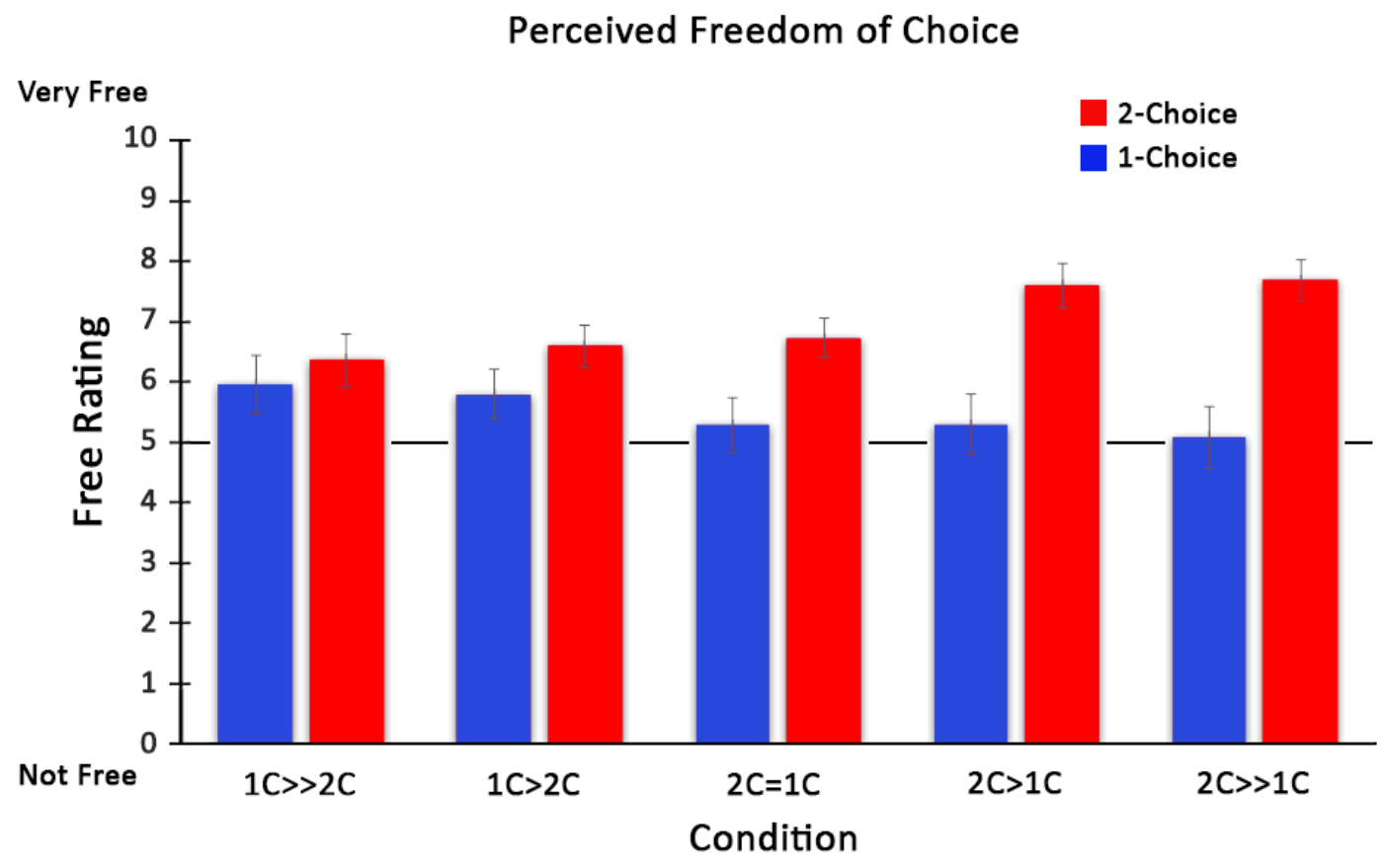

Figure 5. Free ratings given by participants for each room and condition, during Experimental task 1. Free ratings were reported at the end of each block, from 0 (Not Free) to 10 (Very Free). Conditions are indicated by comparative value of the rewards. Choices made in the 2-Choice room were perceived as significantly freer than choices made in the 1-Choice room, where one door was always locked. Gem value also positively influenced perceived freedom, so that choices in each of the rooms were rated significantly more highly as the comparative value of gems in the room increased.

\section{Decision trajectory metrics}

We first aimed to analyse 'changes of mind' demonstrated in the decision trajectories during navigation by considering large-scale $x$-flips. In previous mouse-tracking studies of continuous movement dynamics, $x$-flips have been reported for minor fluctuations in motion trajectories (Barca and Pezzulo, 2015, 2012; Dale et al., 2008). However, in our virtual environment, where movements were smoothly controlled with keyboard button presses, we measured $x$-flips only to detect gross changes of mind in the course of decisions (Figure 6). We analysed $x$-flips that occurred in each of the final rooms with a 2x5 ANOVA. This revealed a main effect of Choice Availability, with flips significantly more common in the 1-Choice room $(M=6.2 \%$, S.E. $=0.9)$ than the 2-Choice room $(M=1.0 \%$, S.E. $=0.3 ; F(1,25)=30.231, p<0.001)$. There was no significant effect of Value and no significant interaction. We repeated this analysis for the first room and found no significant main effects or interactions. Our results thus indicate that participants were more likely to change their intended course of action in the 1-Choice room with the locked door than the 2Choice room and this was independent of the relative reward value. However, overall the frequency of change of mind decisions was very low.

We next analysed the variability in participant trajectories, using the discrete Fréchet distance to measure the average deviation from the mean trajectory in each context (Figure 7). A $2 \times 5$ ANOVA of the trajectories in the final rooms showed a main effect of Choice Availability, whereby there was significantly greater variability in the 2 -Choice room $(M=0.44, S . E .=0.01)$ than the 1 Choice room $(M=0.41$, S.E. $=0.02 ; F(1,25)=6.171, p=0.02)$. We found no significant effect of Value and no significant interactions. Path variability, indicative of choice deliberation, was therefore greater when a second door remained available. A 2x5 ANOVA of trajectories in the first room, in which trials were split according to the final room chosen, showed a significant main effect of Value $(F(4,100)=5.380, p=0.001)$. There was greatest variability evident in the three central conditions, in which the comparative value of the choice was equal or slightly less or more (Table 1). There was no significant effect of Choice Availability in this room and no significant interactions. 
A

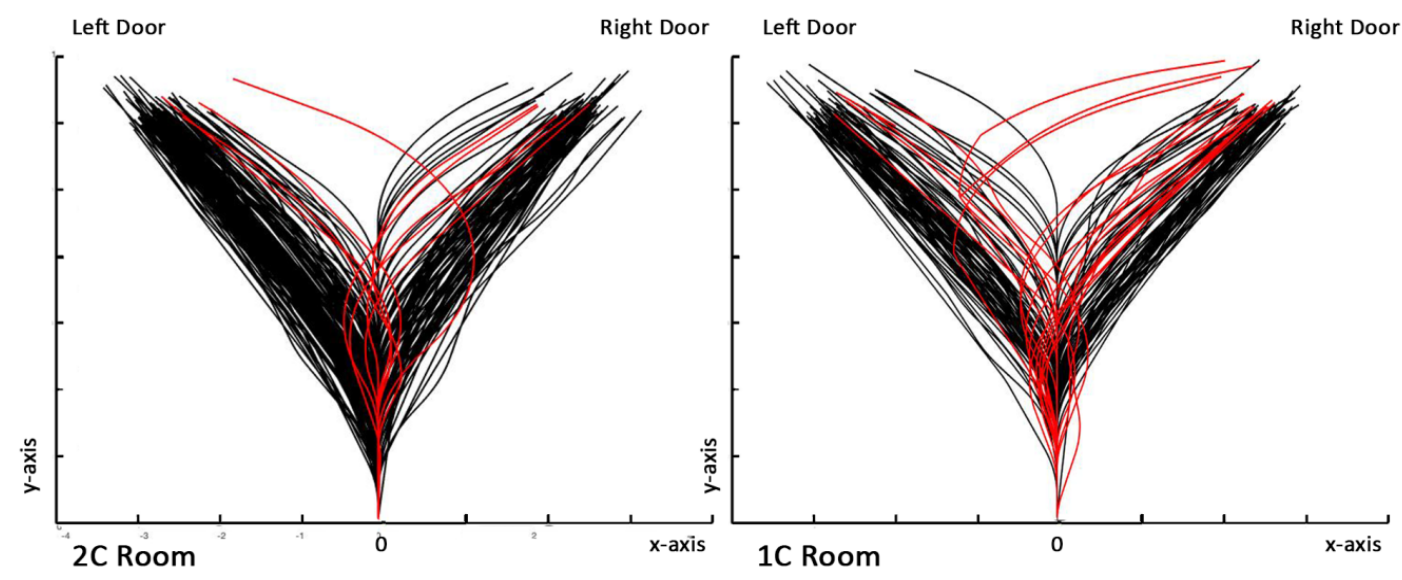

B

Proportion of Flip Trials

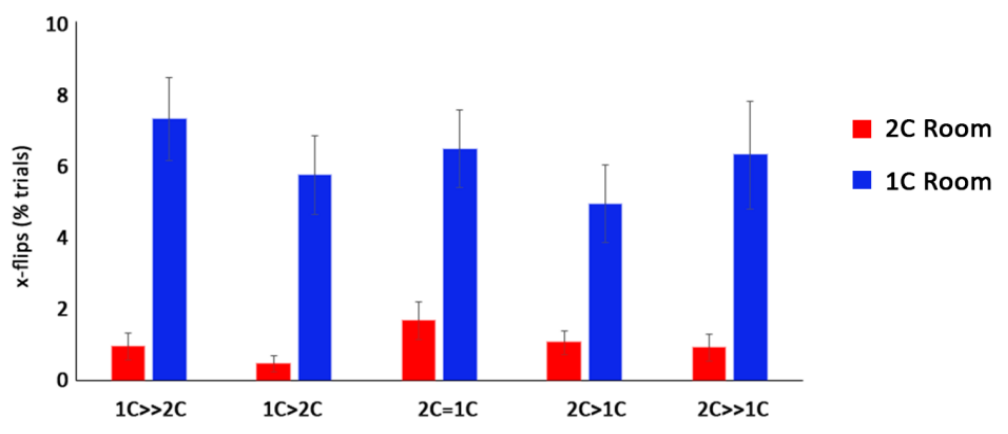

Figure 6. Decision $x$-flips indicate changes of decision during Experimental task 1. A) Decision trajectories for a representative participant are displayed with regular trials in black and x-flips in red for the 2-Choice (left) and 1-Choice (right) rooms. B) x-flips occurred significantly more frequently in the 1-Choice room than the 2-Choice room across all conditions.

\begin{tabular}{cllll} 
VALUE & Mean & S.E. & \multicolumn{2}{c}{$95 \%$ C.I. } \\
Much less & 0.45 & 0.02 & 0.42 & 0.48 \\
Less & 0.50 & 0.01 & 0.47 & 0.52 \\
Equal & 0.48 & 0.02 & 0.45 & 0.51 \\
Greater & 0.47 & 0.01 & 0.44 & 0.50 \\
Much greater & 0.46 & 0.01 & 0.43 & 0.48
\end{tabular}

Table 1. Main effects of Value for trajectory variability during navigation of the first room, towards selection of either of the final rooms, during Experimental task 1. 

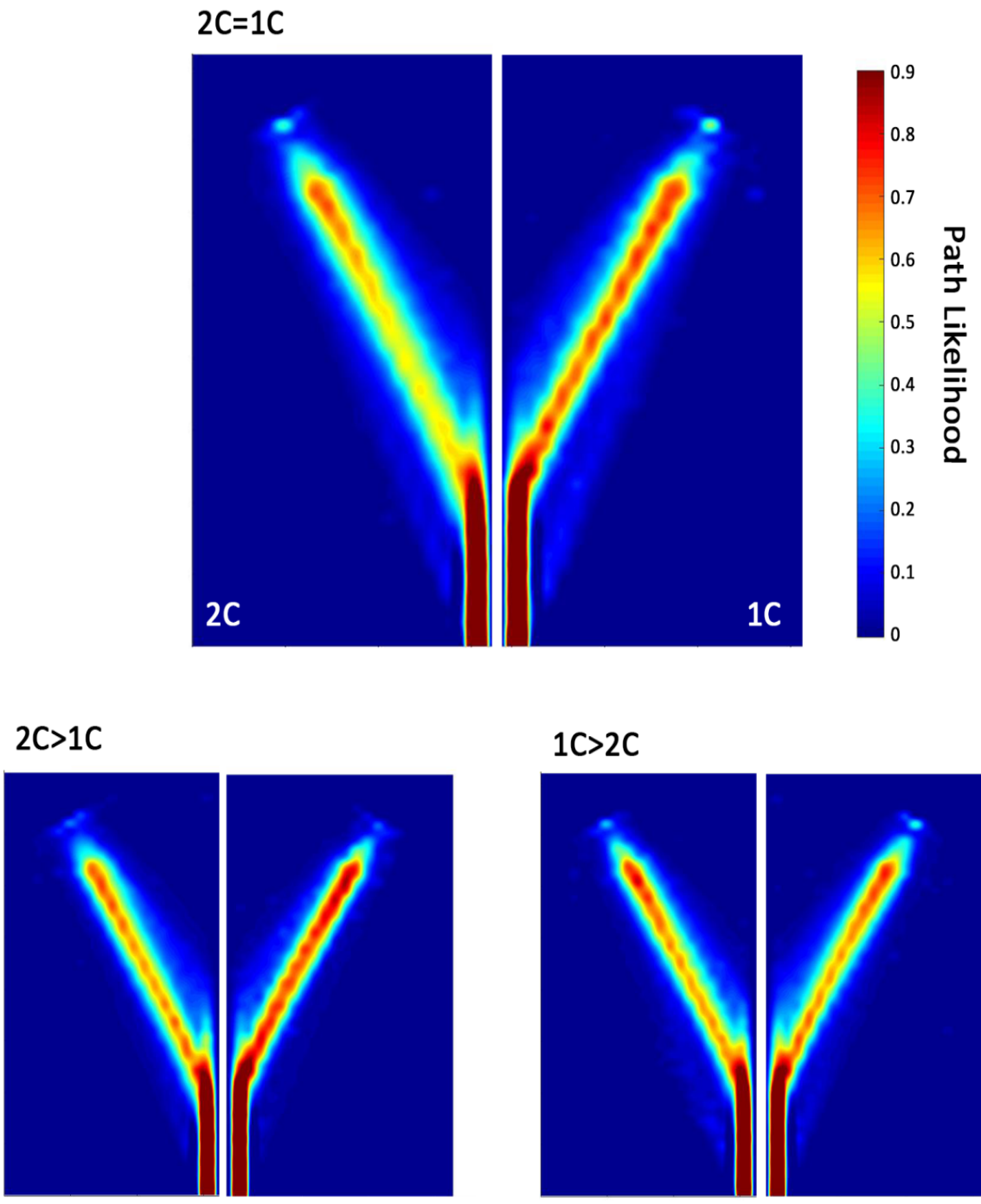

$1 \mathrm{C}>2 \mathrm{C}$
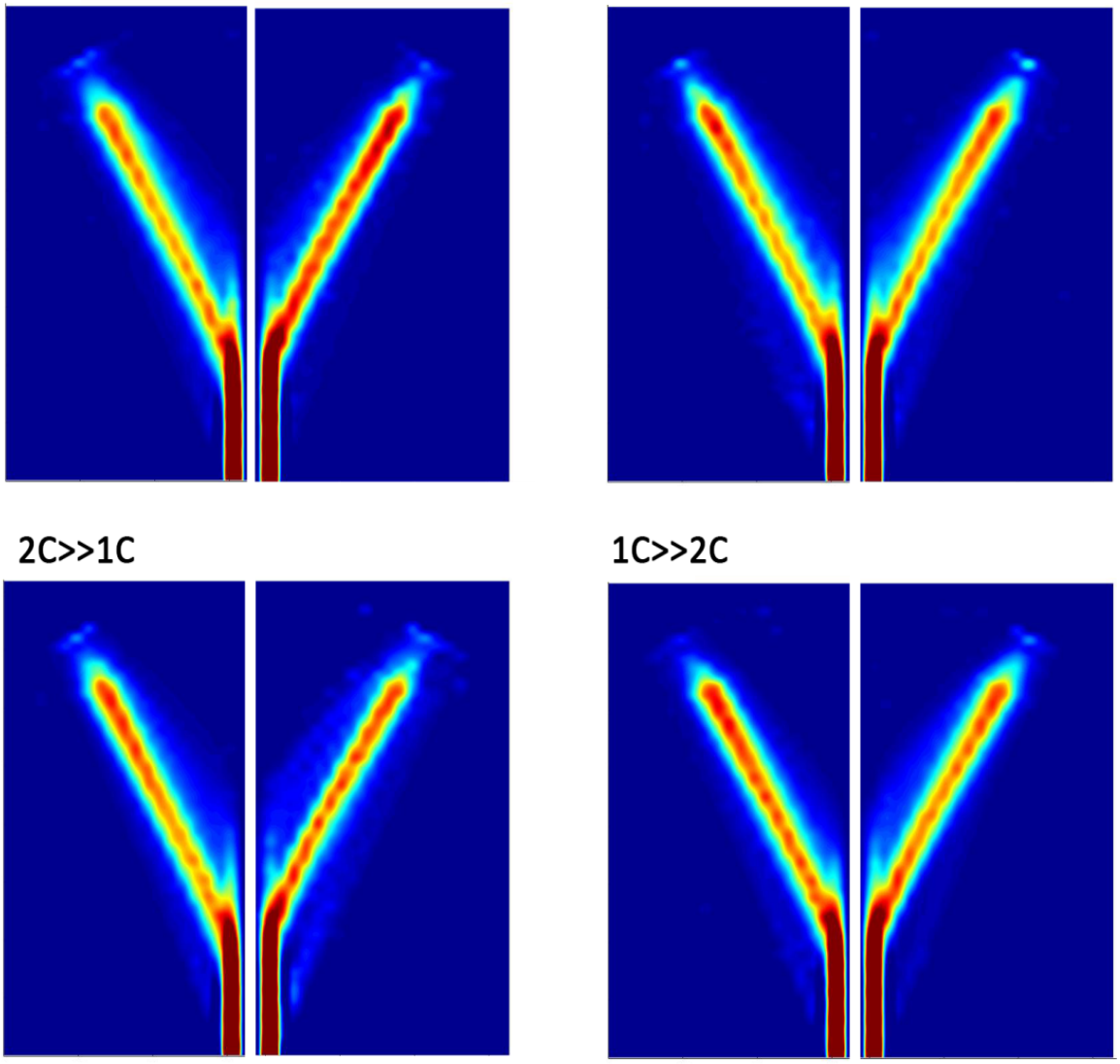

$1 \mathrm{C} \gg 2 \mathrm{C}$

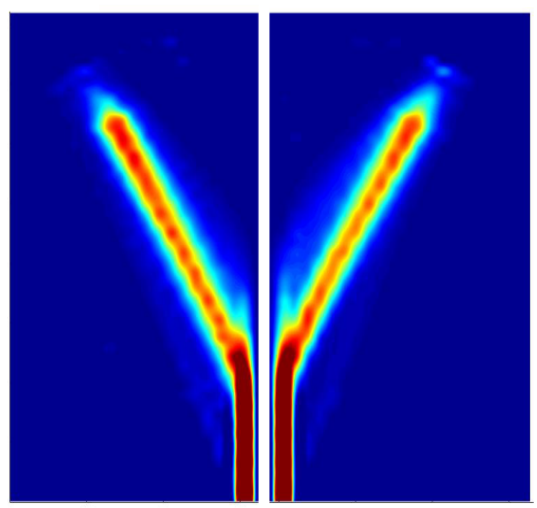

Figure 7. Heat maps showing the common trajectories for the 2-Choice and 1-Choice rooms in each condition, during Experimental task 1. Colour represents the likelihood that each point in coordinate space was crossed on any given trial, giving a visual representation of decision variability. Paths are significantly more variable, based on average Fréchet distances, in the 2-Choice room than the 1-Choice room, which could indicate greater choice deliberation. 


\section{Results of Experimental task 2}

\section{Participant choice behaviour}

We analysed choice proportions in favour of the 4-Choice room over the 3-Choice room across all three conditions of varying relative reward values. In the baseline condition, when all rewards were equal, we found that participants showed a significant choice bias, selecting the 4-Choice room on $59 \%(S E=3 \%$ ) of trials on average (single-sample t-test difference from $50 \%: t(35)=2.822$, $\mathrm{p}=0.008$ ), see Figure $8 \mathrm{~A}$. The overall choice bias when all conditions were analysed collectively was $54 \%(S E=3 \%)$, which does not show significance $(t(35)=1.38, p=0.17)$.

To further examine whether and how many individual participants showed a positive choice bias, we plotted the proportion of times each participant selected the 2-Choice room, see Figure 8B. Overall, many of the participants showed a positive choice bias, as indexed by a proportion greater than 0.5 , but they varied in the choice bias intensity.

Furthermore, we quantified the contributions of utility-seeking to each participant's choice behaviour, to ensure that participants were overall able to select rewarding outcomes. For this, we performed a linear regression analysis to predict participant's choices on each trial on the basis of comparative value. We found that expected utility significantly influenced behaviour (mean $\beta=0.17$, $\mathrm{SE}=0.01, \mathrm{t}(35)=23.92, \mathrm{p}<0.001)$. Individual $\beta$ coefficients for each participant are displayed in Figure $8 \mathrm{C}$. Positive coefficients in the regression models indicated that participant choice behaviour was positively driven by utility-seeking factors. We found that this was the case for all participants. Finally, we asked whether the drive for entropy gain led to a detriment in the reward gained by each participant. We correlated the choice bias for each participant with the $\beta$ coefficient for utility gain and found no relationship $(R=-0.21, p=0.2)$. Hence, entropy-maximising behaviour was not associated with a trade-off in utilitarian gain. 

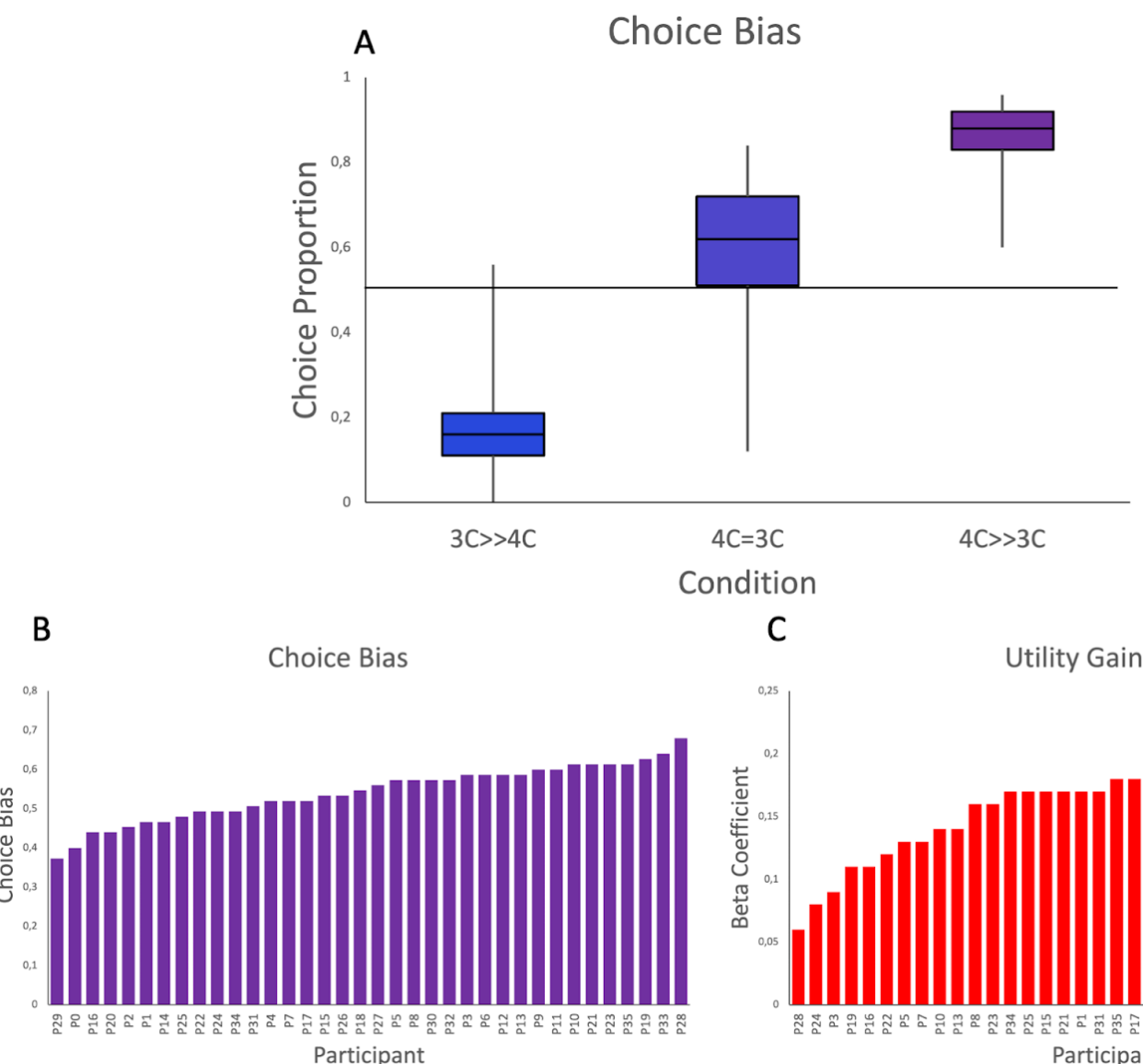

C Utility Gain

Figure 8. Participant choice behavior during Experimental task 2. A) Box-and-whisker plots show distributions of participant choice biases for each condition. Participants showed a significant choice bias, evidenced by a greater proportion of choices for the 2-Choice room than the 1-Choice room. B) Individual choice bias, quantified by the proportion of choices of the 2-Choice room. Almost all participants showed a proportion greater than 0.5, which indexes a positive choice bias. C) Individual linear regression coefficients for the degree to which each participant's choices were predicted by utility gain, sorted in ascending order. Positive coefficients indicate utility-seeking behaviour, whereas negative coefficients indicate avoidance. Participants varied in the extent but, overall, showed a positive tendency for utility.

\section{Subjective free choice ratings}

Average ratings of perceived freedom of choice differed significantly across conditions (Figure 9). A $2 \times 3$ repeated measures ANOVA revealed a main effect of Choice Availability, in which choices made in the 4-Choice room were perceived as freer than those in the 3-Choice room (4Choice: $M=7.47$, SE=0.25; 3-Choice: $M=6.36, S E=0.29 ; F(1,35)=8.198, p=0.005)$. There was no main effect of Value, indicating that the reward available in each room had no influence on perceived freedom. Finally, there was significant interaction between Choice Availability and Value $(F(2,210)=9.486, p<0.001)$. Post-hoc analysis using Tukey Test showed that choices made in the 4-Choice room in the $4 \mathrm{C}>>3 \mathrm{C}$ condition were perceived as freer than those made in the 3-Choice room in the $4 C=3 C$ condition $(p=0.001)$, that in the $4 C>>3 C$ condition, choices made in the 4Choice room were perceived as freer than those made in the 3 -Choice room $(p=0.001)$, that choices made in the 3 -Choice room in the $4 \mathrm{C}<<3 \mathrm{C}$ were perceived as freer than those made in the 3 -Choice room in the $4 C=3 C$ condition $(p=0.019)$, and that choices made in the 4-Choice room in the $4 \mathrm{C}<<3 \mathrm{C}$ condition were perceived as freer than those made in the 4-Choice room in the $4 C>>3 C$ condition $(p=0.02)$.

We also asked whether perceived freedom of choice increases with the number of options available. To answer this question, we considered together the results of Experimental tasks 1 and 2 , which asked the same questions about freedom of choice. We performed (Bonferroni-corrected) t-tests between the 4 conditions of Choice Availability, two (1-Choice and 2-Choice) from 
Experimental tasks 1 and two (3-Choice and 4-Choice) from Experimental tasks 2. The Bonferronicorrected t-tests confirmed that choices made in the 4-Choice room were perceived as freer than those in the 3-Choice room $(p=0.027)$ and that choices made in the 2-Choice room were perceived as freer than those in the 1-Choice room $(p=0.0002)$, as reported above. Furthermore, they showed that that choices made in the 4-Choice room were perceived as freer than those in the 1Choice room $(p=0.000)$. No other comparisons were significant. Fianally, we did not find a linear trend between the 4 conditions of Choice Availability (Pearson's $p=0.13$ ).

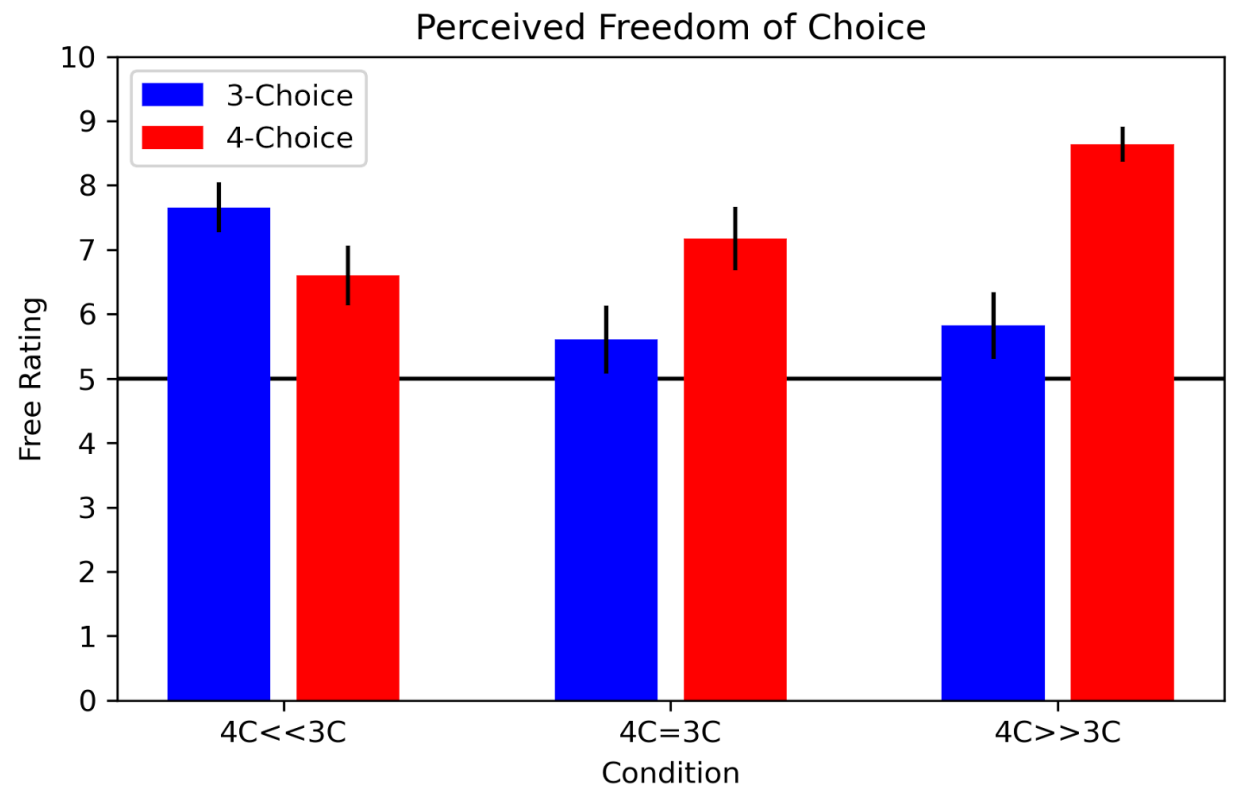

Figure 9. Free ratings given by participants for each room and condition, during Experimental task 2. Free ratings were reported at the end of each block, from 0 (Not Free) to 10 (Very Free). Conditions are indicated by comparative value of the rewards. See the main text for more details.

\section{Decision trajectory metrics}

We aimed to analyse 'changes of mind' and the $x$-flips that occurred in each of the final rooms, with a 2x3 ANOVA. This revealed a main effect of Choice Availability, with flips significantly more common in the $3-$ Choice room $(M=13.7 \%, S . E .=2.1)$ than the 4-Choice room $(M=5.7 \%, S . E .=0.3$; $F(1,35)=13.705, p<0.001)$. There was no significant effect of Value and no significant interaction. We repeated this analysis for the first room and found no significant main effects or interactions. Our results indicate that participants were more likely to change their intended course of action in the 3-Choice room with the locked door than the 4-Choice room and this was independent of the relative reward value. However, overall, the frequency of change of mind decisions was very low.

We next analysed the variability in participant trajectories, using the discrete Fréchet distance to measure the average deviation from the mean trajectory in each context. A 2x3 ANOVA of the trajectories in the final rooms showed a main effect of Choice Availability, with greater variability for the the 3-Choice room $(M=7.25$, S.E. $=0.37)$ compared to the 4-Choice room $(M=6.63, S . E .=0.79$; $F(1,35)=3.958, p=0.047)$. We found no significant effect of Value but significant interaction between Choice Availability and Value $(p<0.001)$. Post-hoc analysis using Tukey Test showed that the trajectory variability in the 4 -Choice room was significantly greater in the $4 \mathrm{C}>>3 \mathrm{C}$ condition compared to the $4 C<<3 C$ condition $(p=0.0004)$; the variability was significantly greater in the 3 Choice compared to the 4-Choice room in the $4 C<<3 C$ condition $(p=0.002)$; and the variability of the 3 -Choice room in the $4 C=3 C$ condition was significantly greater than the 4-Choice room in the $4 C<<3 C$ condition $(p=0.006)$. A $2 \times 3$ ANOVA of trajectories was ran also for the first room, in which trials were split according to the final room chosen: no significant main effect of Value, of Choice Availability and interactions were found. 


\section{Discussion}

We examined free choice behaviour in the context of two sequential choice tasks in which participants navigated through virtual rooms in order to reach reward locations. We report three main findings. First, we found evidence for entropy maximization (i.e., a "choice bias") above and beyond utility maximization during participants' sequential choices. Second, participants reported feeling a greater freedom of choice when more choice options were available. Third, we found that the ongoing consideration of alternative options (when available) was evident in the manner in which participants navigated the virtual environment. We discuss these three findings below.

\section{Evidence for entropy maximization during sequential choices}

We found that during the choice between rooms that gave access to one versus two choices (Experimental task 1) and between rooms that gave access to three versus four choices (Experimental task 2), participants were biased towards the rooms that gave access to more choices - or in other words, the rooms in which they could "keep their options open". In both experimental tasks, the "choice bias" was evident when the rooms to be selected were equally valuable, effectively acting as a choice (entropy) bonus that influenced action selection, above and beyond reward achievement. In Experimental task 1, but not Experimental task 2, the choice bias was also significant across all conditions, when rooms had unbalanced values. The choice bias was not only a group-level phenomenon, but was apparent across almost all participants of Experiment 1 (with the only exception being a few participants that showed no bias) and in the large majority of participants of Experiment 2 (with a few participants showing the opposite bias). Furthermore, the choice bias did not hinder reward-seeking behaviour. Taken together, these results support models of decision-making in which utility and entropy are optimized together, rather than classical models that focus exclusively on utility maximization. To gather extra support for this idea, we implemented an inference-based computational model that uses both utility and entropy and showed that it better accounts for choice behaviour (in Experimental task 1) than a model based on expected utility alone. Notably, based on our model parameterisation, the degree of choice bias predicted by the inference model of the task very closely matched the observed participant bias. Therefore, the inclusion of entropy maximisation could account for choice behaviour in situations where a drive for utilitarian value alone fails to explain observations.

Choice bias has classically been reported using a choice preference task, in which participants choose between one action that leads directly to an outcome or an action that provides further choice (Bown et al., 2003; Catania, 1975; Fujiwara et al., 2013; Leotti and Delgado, 2011; Ono, 2000; Suzuki, 1999, 1997). These studies have typically reported a bias of approximately twothirds, whereby participants selected the choice providing two options twice as often as the choice leading to only one option. We first wanted to ensure that this classical finding was not the result of participants equally sampling each of the three possible outcomes. To address this, our tasks were designed with four (Experimental task 1) or eight (Experimental task 2) final goal states overall but with the comparative number of available options conserved on each trial, specifically two options versus one (Experimental task 1) or four options versus three. If the choice bias previously reported was driven only by equal selection of each outcome state, the proportion of choices for the 2-Choice room (Experimental task 1) or the 4-Choice room (Experimental task 2) would be 0.5 whereas preference for choice would result in a positive bias. We found that choice was preferred in both Experimental tasks, as evident in the shift towards choosing the room with more doors available. Thus, we found similar bias to previous studies even when accounting for differences in the number of goal outcomes, which provides strong support for the observation that participants seek contexts that provide choice.

A previous study by Schwartenbeck et al. (2015) reported a preference for choice in a binary choice paradigm in which participants could chose between two offers containing between one and four different snacks - and knew that one of the snacks of the selected offer would have been selected randomly and possibly received at the end of the study. The study found that participants preferred options that provided a higher numbers of items, even when choosing the smaller set would have led to an increased likelihood of attaining the most valuable item. Furthermore, similar 
to our study, participants' choice behavior could be explained more accurately with an inferencebased model that considers both utility and entropy than expected utility theory alone. However, while this study supports the idea that preference for choice can be accounted for with an inferential framework, it addressed only a simple one-step choice scenario in which the selection of high entropy states had no influence on the availability of future choices. Rather, choices in the task resulted in one outcome on completion of the task that was taken at random from a set, meaning that entropy maximisation did not consequentially affect the outcomes attained. Furthermore, the study of Schwartenbeck et al. (2015) could not fully disentangle whether preference for future options is related to entropy maximisation, as opposed to simply resulting from equal sampling of each end state. Specifically, the reported choice bias of approximately twothirds has corresponded to the choice leading to two options being selected twice as often as the choice leading to one option. Our study addresses the above limitations by using a sequential choice design, in which the first choice translates into more or less subsequent choice availability (rather than to the random selection of one outcome) and by controlling for the number of possible states to be explored (which are the same in the 1-Choice and 2-Choice rooms and in the 3Choice and 4-Choice rooms), therefore providing additional support for the idea that entropy maximization is a significant consideration in guiding ongoing choice behaviour.

Preference for choice has been proposed to exist as an inherent drive that motivates an agent to seek optimal states in the environment (Leotti et al., 2010; Leotti and Delgado, 2011). Similarly, inference-based models suggest that agents are driven to select high entropy states as a means of maximising the likelihood of expected outcomes (Friston, 2010; Pezzulo et al., 2015), or optimising decision-making without requiring a laborious, and often unfeasible, assessment of the values of all available actions (Schwartenbeck et al., 2015; Volz and Gigerenzer, 2012). This is particularly crucial in real-world environments, where the availability of options changes over time (Cisek and Kalaska, 2010; Klyubin et al., 2008; Lepora and Pezzulo, 2015). Maximising for entropy as well as utility may therefore serve to enhance the adaptive control an agent maintains over future outcomes as it navigates an uncertain world (Klyubin et al., 2008; Lancia et al., 2022; Pezzulo and Ognibene, 2011; Rick and Loewenstein, 2008; Schwartenbeck et al., 2015). In our task, participants experienced a simplified version of navigating a virtual world with uncertainty. Our findings support these theories by demonstrating a bias for higher entropy states, which however does not hinder reward-seeking, therefore offering a novel perspective that accounts for choice preference as a means of optimising for the availability of future possibilities.

One question raised by our study regards the differences between some of the results of the two experiments. In both experiments, we found a choice bias in the case of two rooms with the same (average) value. However, we only found an overall choice bias across all conditions in the first experiment. On the one hand, the fact that the choice bias is present in the most discriminative condition (that is, when entropy maximization is not confounded by utility considerations) is reassuring. On the other hand, the difference between the two experiments suggests that entropy and utility factors might interact in complex ways to determine choices or that the availability of a single choice (i.e., the 1-Choice room in Experimental task 1) should be considered as a special case, in which considerations of entropy are more prominent. These and other hypotheses remain to be tested in future studies.

Another research question regards the possibility that states with excessive levels of entropy and choice availability could be less preferred rather than more preferred, as found in our study. Previous research has demonstrated that too many choices could provide a choice overload, avoidance and loss of motivation (lyengar and Lepper, 2000) but it is unclear what is the point at which choices become excessive and how to integrate the preference for choice and choice overload in the same computational model.

\section{Greater freedom of choice with more choice options available}

In both Experimental Tasks 1 and 2, we found that the availability of choice significantly influenced participants' perceived freedom of choice, whereas reward value significantly influenced perceived freedom of choice only in Experimental task 1. The fact that in both experiments participants reported having more freedom of choice in rooms where more options were available 
supports the idea that the feeling of having free will could be intimately related to choice availability. Further support for this idea comes from the fact that, when combining the results of both experiments (which used the same experimental questions), we found that choices made in the 4-Choice room were perceived as freer than those in the 1-Choice room. However, our results suggest that choice availability does not scale linearly with the number of choices, hence their specific relations remain to be investigated in future studies.

Availability of choice has long been a central theme in modern views of free will (Banja, 2015; Monroe and Malle, 2009). Our results further show that in both Experimental tasks, participants not only preferentially chose states with greater choice availability, but reported higher perceived freedom in this context. This is in accordance with previous findings suggesting that the subjective experience of free choice corresponds to the availability of an alternative option (Rens et al., 2018) or the perception of having a higher number of options available (Filevich et al., 2013). We also found that in Experimental task 1 (but not Experimental task 2), comparative reward value positively influenced perceived freedom. Libertarian views of free will emphasise the ability to choose any available option and, therefore, regard reward as a negative constraint on the freedom an individual has to choose (Schultz, 2015). However, others have indicated that the value of outcomes influences the perception of freedom during free choice; for example participants reported being freer when choosing between two equally good than two equally bad options (Lau et al., 2015; Lau and Wenzel, 2015). Our finding of a positive relationship between the value of decision outcomes and subjective freedom could lend some support the latter view; however, given that this relationship was only apparent in Experimental task 1 suggests that more evidence is needed to adjudicate between the alternative views.

\section{Choice availability influences ongoing decision dynamics}

As we recorded (virtual) path trajectories during the choice, our experiments permit asking how choice availability influences participants' ongoing decision dynamics. We found that in both Experimental Tasks 1 and 2, participants were more likely to change their decision course in the rooms with the locked doors; but note that such 'changes of mind' were rare. The most likely explanation for this is that, at least on occasion, participants forward-planned the final choice prior to entering the second room, and subsequently had to change course upon discovering that their chosen door was locked. This finding would have been unlikely had participants made two independent sequential choices, waiting to enter the second room before choosing left or right again. Alternatively, participants either did not readily perceive which door was locked or did not pay attention to the room orientation. However, this is not implied by other findings such as the choice bias and the good performance of participants. Both reinforcement learning and inferencebased models propose that participants rely on forward planning (Botvinick and Toussaint, 2012; Daw et al., 2011; Pezzulo et al., 2017, 2014; Pezzulo and Ognibene, 2011; Redish, 2016; Simon and Daw, 2011; Stoianov et al., 2022), but this has been challenging to evidence behaviourally. Our findings suggest that participants could have planned for the final goal location in advance, but maintained a malleable state of decision preparation and revised their plan as necessary.

A further indication of malleability in decision-making comes from the finding that participants' path trajectories in Experimental task 1 showed more variability when an alternative option remained available (i.e., in the 2-Choice room) compared to the case of no alternatives (i.e., in the 1-Choice room), indicating ongoing choice consideration during the trial. In Experimental task 1, path variability was also greatest in the first room when the comparative value of each of the subsequent rooms was equal or relatively similar, thus rendering the choice more uncertain and difficult. As the door leading to reward was only reached at the end of the subsequent decision, this suggests participants actively deliberated access to future reward during preparation of their immediate actions. However, we found that path variability is not a simple function of the number of choices available. The results of Experimental task 2 indicate that participants show greater movement variability in the (3-Choice) room that provides fewer options. Yet, it is important to note that different from Experiment 1, in Experiment 2 both rooms gave access to multiple options, therefore affording continuous deliberation. Therefore, while the two experiments suggest that choice availability (versus not availability) promotes greater variability of movement, it remains to 
be fully assessed how the variability depends on various factors, such as the number of options, their values, their geometric arrangement, or others.

The above findings resonate well with numerous other studies tracking decision-making dynamics that report that choices are not completed before initiating an action, and that movements become more variable as a function of choice uncertainty (Dale et al., 2008, 2008; Flumini et al., 2015; Freeman et al., 2011; McKinstry et al., 2008; Pezzulo et al., 2018; Song and Nakayama, 2009). Embodied theories of cognition propose that all available action plans are proactively primed, permitting an agent to act adaptively in the environment even before full evidence towards one action is gathered (Cisek, 2007; Cisek and Kalaska, 2010; Lepora and Pezzulo, 2015; Pezzulo and Cisek, 2016; Stanford et al., 2010). The flexibility of preparation has been also suggested to be a hallmark of free decisions (Fleming et al., 2009; Salvaris and Haggard, 2014). In keeping, a previous study has shown that, while free choices can be predicted from neural activity, neural encoding of options and visual attention both indicate that alternative options remain online during free choice preparation (Rens et al., 2018). Finally, delaying commitment to a particular outcome may also allow an individual greater time to accrue evidence, particular in dynamic contexts (Bown et al., 2003; Lepora and Pezzulo, 2015; Pezzulo and Ognibene, 2011; Weld, 1994). In our Experimental tasks, there was no objective difference in the reward that could be gained from either door but, nevertheless, participants may have sought to optimise their outcomes based on perceived trial-to-trial changes in valuation. In sum, actively seeking states of higher entropy might constitute an adaptive strategy in ecological conditions.

There are a few considerations to take into account in this study. First, the tasks were designed to ensure that there were four (or eight) goal locations for participants to choose between. This was important to verify that any choice bias reported was not the result of equal outcome sampling. Our participants' behaviour suggests that they did plan ahead for the final doors but, as a result, appear to have been forced to occasionally change decisions when a door was locked. This scenario is not equivalent to a single option existing, as avoidance of the locked door (or skull cue hidden behind in Experimental task 1), could arguably have been perceived as an external constraint to a planned choice. A possibility, therefore, is that the perceived cognitive load of having to choose the one available door in the 1-Choice room (or the 3-Choice room) was experienced as a higher cost, or inverse value, leading the 2-Choice room (or the 4-Choice room) to be more preferable. The inference-based entropy maximisation may then also be interpreted as participants acting according to optimal cost-to-go policies (Todorov, 2009). Nevertheless, subjective reports did not indicate an absence of perceived freedom in the 1-Choice or the 3Choice rooms, which we would have expected if participants felt negatively about this state or unable to make their desired choice. Second, the model we built is a simplified version of the task environment, which considers the key factor of choice availability but does not take into account any behavior related to learning or trial-to-trial reward variance. Therefore, this model could be further improved in the future to more fully account for decision behaviour.

In summary, in two experiments, we found evidence of entropy maximisation in free choice behaviour, suggesting that individuals are driven to maximise entropy in addition to utility, by actively seeking conditions in which several options are available. Findings of choice preference have previously been difficult to reconcile with purely economic theories of decision-making. The present results provide a novel perspective accounting for choice bias as entropy gain within an inferential perspective of voluntary decision-making. Tracking the time course of the decision suggests that participants used forward planning to make two consecutive decisions, while at the same time demonstrating flexibility in ongoing option consideration. Finally, we found that choice availability and (in one experiment) utility promoted a subjective sense of free will. This raises the possibility that the subjective experience of free choice could be related to a proactive process of inference of action possibilities and their outcomes. Taken together, our results from behavioural, physical, and subjective measures emphasise the central role proactive choice plays in free decisions and contribute to a theory of free choice behaviour as optimal decision-making in a dynamic world. 


\section{Acknowledgments}

This study was supported by funding from the University of Queensland to NR and RC; the European Union's Horizon 2020 Framework Programme for Research and Innovation under the Specific Grant Agreement No. 785907 and 945539 (Human Brain Project SGA2 and SGA3) to GP; and the European Research Council under the Grant Agreement No. 820213 (ThinkAhead) to GP. The authors declare that the research was conducted in the absence of any commercial or financial relationships that could be construed as a potential conflict of interest. The authors are grateful to their sources of funding, Dr David Lloyd for invaluable technical support, and colleagues in Cunnington Lab for insightful discussions.

\section{Datasets}

The dataset of participant files of Experimental task 1 is available for access on the Harvard Dataverse at https://doi.org/10.7910/DVN/EKSVXE. The files include individual participant .mat files, group .mat files for behaviour, free ratings, motion-tracking data, and a readme to explain structure. The reference for this dataset is (Rens 2019). The dataset of participant files of Experimental task 2 is available for access on the Open Science Framework (OSF) at https://osf.io/jcrsd/. The files include a .json file with all the experimental data and a .xlsx file with all the demographic information. The reference for this dataset is (Lancia 2022). 


\section{References}

Banja J. 2015. "Could Have Chosen Otherwise Under Identical Conditions": An Evolutionary Perspective on Free Will. AJOB Neuroscience 6:3-11. doi:10.1080/21507740.2015.1014071

Barca L, Pezzulo G. 2015. Tracking Second Thoughts: Continuous and Discrete Revision Processes during Visual Lexical Decision. PLOS ONE 10:e0116193. doi:10.1371/journal.pone.0116193

Barca L, Pezzulo G. 2012. Unfolding Visual Lexical Decision in Time. PLoS ONE.

Bennett D, Bode S, Brydevall M, Warren H, Murawski C. 2016. Intrinsic Valuation of Information in Decision Making under Uncertainty. PLoS Comput Biol 12:e1005020. doi:10.1371/journal.pcbi.1005020

Botvinick M, Toussaint M. 2012. Planning as inference. Trends in Cognitive Sciences 16:485-488. doi:10.1016/j.tics.2012.08.006

Bown NJ, Read D, Summers B. 2003. The lure of choice. Journal of Behavioral Decision Making 16:297-308. doi:10.1002/bdm.447

Catania AC. 1975. Freedom and knowledge: an experimental analysis of preference in pigeons. $J$ Exp Anal Behav 24:89-106. doi:10.1901/jeab.1975.24-89

Cisek P. 2007. Cortical mechanisms of action selection: the affordance competition hypothesis. Phil Trans R Soc B 362:1585-1599.

Cisek P, Kalaska JF. 2010. Neural mechanisms for interacting with a world full of action choices. Annu Rev Neurosci 33:269-298. doi:10.1146/annurev.neuro.051508.135409

Cockburn J, Collins AG, Frank MJ. 2014. A reinforcement learning mechanism responsible for the valuation of free choice. Neuron 83:551-7. doi:10.1016/j.neuron.2014.06.035

Dale R, Roche J, Snyder K, McCall R. 2008. Exploring action dynamics as an index of pairedassociate learning. PLoS One 3:e1728. doi:10.1371/journal.pone.0001728

Daw ND, Gershman SJ, Seymour B, Dayan P, Dolan RJ. 2011. Model-based influences on humans' choices and striatal prediction errors. Neuron. 69(6):1204-15. doi: 10.1016/j.neuron.2011.02.027

Eiter T, Mannila H. 1994. Computing discrete Fréchet distance.

Filevich E, Vanneste P, Brass M, Fias W, Haggard P, Kuhn S. 2013. Brain correlates of subjective freedom of choice. Conscious Cogn 22:1271-84. doi:10.1016/j.concog.2013.08.011

Fleming SM, Mars RB, Gladwin TE, Haggard P. 2009. When the brain changes its mind: flexibility of action selection in instructed and free choices. Cereb Cortex 19:2352-2360. doi:10.1093/cercor/bhn252

Flumini A, Barca L, Borghi AM, Pezzulo G. 2015. How do you hold your mouse? Tracking the compatibility effect between hand posture and stimulus size. Psychological research 79:928-938.

Fréchet MM. 1906. Sur quelques points du calcul fonctionnel. Rend Circ Matem Palermo 22:1-72. doi:10.1007/BF03018603

Freeman JB, Dale R, Farmer TA. 2011. Hand in motion reveals mind in motion. Front Psychol 2:59. doi:10.3389/fpsyg.2011.00059

Friston K. 2010. The free-energy principle: a unified brain theory? Nat Rev Neurosci 11:127-138. doi:10.1038/nrn2787

Friston K, Schwartenbeck P, FitzGerald T, Moutoussis M, Behrens T, \& Dolan RJ. 2013. The anatomy of choice: active inference and agency. Frontiers in human neuroscience 7:598.

Friston K, FitzGerald T, Rigoli F, Schwartenbeck P, Pezzulo G. 2017. Active Inference: A Process Theory. Neural Comput 29:1-49. doi:10.1162/NECO_a_00912

Friston K, Rigoli F, Ognibene D, Mathys C, Fitzgerald T, Pezzulo G. 2015. Active inference and epistemic value. Cognitive Neuroscience 0:1-28. doi:10.1080/17588928.2015.1020053

Fujiwara J, Usui N, Park SQ, Williams T, lijima T, Taira M, Tsutsui K, Tobler PN. 2013. Value of freedom to choose encoded by the human brain. $J$ Neurophysiol 110:1915-29. doi:10.1152/jn.01057.2012 
lyengar, S.S. and Lepper, M.R., 2000. When choice is demotivating: Can one desire too much of a good thing? Journal of personality and social psychology, 79(6), p.995.

Inesi ME, Botti S, Dubois D, Rucker DD, Galinsky AD. 2011. Power and choice: their dynamic interplay in quenching the thirst for personal control. Psychol Sci 22:1042-8. doi:10.1177/0956797611413936

Jaynes ET. 1957. Information theory and statistical mechanics. II. Physical review 108:171.

Kahneman D, Tversky A. 1979. Prospect Theory: An Analysis of Decision under Risk. Econometrica 47:263-292.

Klyubin AS, Polani D, Nehaniv CL. 2008. Keep your options open: an information-based driving principle for sensorimotor systems. PLoS One 3:e4018. doi:10.1371/journal.pone.0004018

[DATASET] Lancia GL. 2022. Replication Data for: Evidence for entropy maximisation in human free choice behaviour, Experimental task 2, https://osf.io/jcrsd/, Open Science Framework

Lancia GL, Eluchans M, D'Alessandro M, Spiers H, Pezzulo G. 2022. Humans account for cognitive costs when finding shortcuts: An information-theoretic analysis of navigation. bioRxiv preprint

Lau S, Hiemisch A, Baumeister RF. 2015. The experience of freedom in decisions - Questioning philosophical beliefs in favor of psychological determinants. Conscious Cogn 33:30-46. doi:10.1016/j.concog.2014.11.008

Lau S, Wenzel M. 2015. The effects of constrained autonomy and incentives on the experience of freedom in everyday decision-making. Philosophical Psychology 28:967-979. doi:10.1080/09515089.2014.951718

Leotti LA, Delgado MR. 2011. The inherent reward of choice. Psychol Sci 22:1310-8. doi:10.1177/0956797611417005

Leotti LA, lyengar SS, Ochsner KN. 2010. Born to choose: the origins and value of the need for control. Trends Cogn Sci 14:457-63. doi:10.1016/j.tics.2010.08.001

Lepora NF, Pezzulo G. 2015. Embodied Choice: How action influences perceptual decision making. PLoS Comput Biol.

McKinstry C, Dale R, Spivey MJ. 2008. Action dynamics reveal parallel competition in decision making. Psychol Sci 19:22-24. doi:10.1111/j.1467-9280.2008.02041.x

Monroe AE, Malle BF. 2009. From Uncaused Will to Conscious Choice: The Need to Study, Not Speculate About People's Folk Concept of Free Will. Review of Philosophy and Psychology 1:211-224. doi:10.1007/s13164-009-0010-7

Nash JF. 1950. The Bargaining Problem. Econometrica 18:155-162. doi:10.2307/1907266

Ono null. 2000. Free-choice preference under uncertainty. Behav Processes 49:11-19.

Parr T., Pezzulo G., Friston K. (2022) Active Inference. The Free Energy Principle in Mind, Brain, and Behavior. MIT Press

Pattanaik PK, Xu Y. 1998. On Preference and Freedom. Theory and Decision 44:173-198. doi:10.1023/A:1004924211553

Pezzulo G, Cartoni E, Rigoli F, Pio-Lopez L, Friston K. 2016. Active Inference, epistemic value, and vicarious trial and error. Learn Mem 23:322-338. doi:10.1101//m.041780.116

Pezzulo G, Cisek P. 2016. Navigating the Affordance Landscape: Feedback Control as a Process Model of Behavior and Cognition. Trends in Cognitive Sciences 20:414-424. doi:10.1016/j.tics.2016.03.013

Pezzulo G., lodice P, Barca L, Chausse P, Monceau S, Mermillod M. 2018. Increased heart rate after exercise facilitates the processing of fearful but not disgusted faces. Scientific Reports 8:398. doi:10.1038/s41598-017-18761-5

Pezzulo G, Kemere C, Meer M van der. 2017. Internally generated hippocampal sequences as a vantage point to probe future-oriented cognition. Annals of the New York Academy of Sciences 1396:144-165.

Pezzulo G, Ognibene D. 2011. Proactive Action Preparation: Seeing Action Preparation as a Continuous and Proactive Process. Motor Control.

Pezzulo G, Rigoli F, Friston K. 2018. Hierarchical Active Inference: A Theory of Motivated Control. Trends in Cognitive Sciences 22:294-306. doi:10.1016/j.tics.2018.01.009 
Pezzulo G, Rigoli F, Friston KJ. 2015. Active Inference, homeostatic regulation and adaptive behavioural control. Progress in Neurobiology 136:17-35.

Pezzulo G, van der Meer MAA, Lansink CS, Pennartz CMA. 2014. Internally generated sequences in learning and executing goal-directed behavior. Trends in Cognitive Sciences 18:647657. doi:10.1016/j.tics.2014.06.011

[DATASET] Rens N. 2019. Replication Data for: Evidence for entropy maximisation in human free choice behaviour, https://doi.org/10.7910/DVN/EKSVXE, Harvard Dataverse, V1

Redish AD. 2016. Vicarious trial and error. Nat Rev Neurosci 17:147-159. doi:10.1038/nrn.2015.30

Rens N, Bode S, Cunnington R. 2018. Perceived freedom of choice is associated with neural encoding of option availability. Neurolmage 177:59-67.

Resulaj A, Kiani R, Wolpert DM, Shadlen MN. 2009. Changes of mind in decision-making. Nature 461:263-266. doi:10.1038/nature08275

Rick S, Loewenstein G. 2008. Intangibility in intertemporal choice. Philos Trans $R$ Soc Lond B Biol Sci 363:3813-24.

Rigoux L, Stephan KE, Friston KJ, Daunizeau J. 2014. Bayesian model selection for group studies - revisited. Neuroimage 84:971-85. doi:10.1016/j.neuroimage.2013.08.065

Roskies AL. 2010. How does neuroscience affect our conception of volition? Annu Rev Neurosci 33:109-30. doi:10.1146/annurev-neuro-060909-153151

Ryan RM, Deci EL. 2000. Intrinsic and Extrinsic Motivations: Classic Definitions and New Directions. Contemp Educ Psychol 25:54-67. doi:10.1006/ceps.1999.1020

Salvaris M, Haggard P. 2014. Decoding intention at sensorimotor timescales. PLoS One 9:e85100. doi:10.1371/journal.pone.0085100

Schultz W. 2015. Neuronal Reward and Decision Signals: From Theories to Data. Physiol Rev 95:853-951. doi:10.1152/physrev.00023.2014

Schwartenbeck P, FitzGerald TH, Mathys C, Dolan R, Kronbichler M, Friston K. 2015. Evidence for surprise minimization over value maximization in choice behavior. Sci Rep 5:16575. doi:10.1038/srep 16575

Schwartenbeck P, Friston K. 2016. Computational Phenotyping in Psychiatry: A Worked Example. eNeuro 3. doi:10.1523/ENEURO.0049-16.2016

Schwartenbeck P, Passecker J, Hauser T, FitzGerald THB, Kronbichler M, Friston KJ. 2018. Computational mechanisms of curiosity and goal-directed exploration. bioRxiv 411272 . doi:10.1101/411272

Simon DA, Daw ND. 2011. Neural correlates of forward planning in a spatial decision task in humans. J Neurosci 31:5526-5539. doi:10.1523/JNEUROSCI.4647-10.2011

Song J-H, Nakayama K. 2009. Hidden cognitive states revealed in choice reaching tasks. Trends Cogn Sci 13:360-366. doi:10.1016/j.tics.2009.04.009

Spivey MJ, Dale R. 2006. Continuous Dynamics in Real-Time Cognition. Curr Dir Psychol Sci 15:207-211. doi:10.1111/j.1467-8721.2006.00437.x

Stanford TR, Shankar S, Massoglia DP, Costello MG, Salinas E. 2010. Perceptual decision making in less than 30 milliseconds. Nat Neurosci 13:379-385. doi:10.1038/nn.2485

Stephan KE, Penny WD, Daunizeau J, Moran RJ, Friston KJ. 2009. Bayesian Model Selection for Group Studies. Neuroimage 46:1004-1017. doi:10.1016/j.neuroimage.2009.03.025

Stoianov I, Maisto D, Pezzulo G. 2022. The hippocampal formation as a hierarchical generative model supporting generative replay and continual learning. Progress in Neurobiology, 217, 102329

Stillman TF, Baumeister RF, Mele AR. 2011. Free will in everyday life: Autobiographical accounts of free and unfree actions. Philosophical Psychology 24:381-394. doi:10.1080/09515089.2011.556607

Suzuki S. 1999. Selection of Forced- and Free-Choice by Monkeys (Macaca Fascicularis). Percept Mot Skills 88:242-250. doi:10.2466/pms.1999.88.1.242

Suzuki S. 1997. Effects of number of alternatives on choice in humans. Behavioural Processes 39:205-214. doi:10.1016/S0376-6357(96)00049-6 
Todorov E. (2009). Efficient computation of optimal actions. Proceedings of the National Academy of Sciences. 106(28) 11478-11483; DOI: 10.1073/pnas.0710743106

Volz KG, Gigerenzer G. 2012. Cognitive Processes in Decisions Under Risk are not the Same as in Decisions Under Uncertainty. Front Neurosci 6:105. doi:10.3389/fnins.2012.00105

Weld DS. 1994. An Introduction to Least-Commitment Planning. Al Magazine 15:27-61. 